\title{
Asymptotic Stability Criteria for a Class of Impulsive Functional Differential Systems
}

\author{
Martin J. Bohner ${ }^{1, *}$ and Ivanka M. Stamova ${ }^{2}$ \\ ${ }^{1}$ Department of Mathematics and Statistics, Missouri S\&T, 400 West 12th Street, Rolla, MO 65409-0020, USA \\ ${ }^{2}$ Department of Mathematics, University of Texas at San Antonio, One UTSA Circle, San Antonio, TX 78249, USA
}

Received: 2 Jul. 2013, Revised: 4 Oct. 2013, Accepted: 5 Oct. 2013

Published online: 1 Jul. 2014

\begin{abstract}
In this paper, a class of impulsive functional differential systems is investigated. It is proved that for the asymptotic stability of the zero solution of the system considered, it is sufficient that only some components of the right-hand side of the system are bounded for unbounded values of time. For functional differential equations without impulses, similar results were proved by Burton and Makay using Lyapunov-Krasovskii functionals. The goal of this paper is to prove these criteria for a class of impulsive functional differential systems with variable impulsive perturbations applying the Lyapunov-Razumikhin technique.
\end{abstract}

Keywords: Asymptotic stability, Lyapunov-Razumikhin function, impulsive functional differential equations

\section{Introduction}

Since the 1960s, different classes of functional differential equations (FDEs) have been object of numerous investigations related to the applications of these equations to almost every area of applied sciences $[3,5,6]$. The framework of the more than 50 years old stability theory and asymptotic behavior studies for FDEs have not lost their attraction and have been extended widely. On the other hand, many physical systems undergo abrupt changes at certain moments of time due to impulsive inputs. In terms of the mathematical treatment, the presence of pulses gives the system a mixed nature, both continuous and discrete [1, 2, 4, 7-9]. At the present time, there have appeared many results for equations with fixed moments of impulse effect. In the investigation of impulsive differential equations with variable impulsive perturbations, there arises a number of difficulties related to the phenomenon "beating" of the solutions, bifurcation, loss of the property of autonomy, etc. $[2,7,8]$. The wider application, however, of this type of equations requires the formulation of effective criteria for stability of their solutions. In the present paper, the asymptotic stability of the zero solution for a class of impulsive functional differential systems with variable impulsive perturbations is studied. For this purpose, piecewise continuous auxiliary functions are used which are an analogue of Lyapunov functions. Moreover, the technique of investigation essentially depends on the choice of minimal subsets of a suitable space of piecewise continuous functions, by the elements of which the derivatives of Lyapunov's functions are estimated. The method is known as the Lyapunov-Razumikhin function method [6-9]. It is proved that for the asymptotic stability of the zero solution of the system considered, it is sufficient that only some components of the right-hand side of the system are bounded for unbounded values of $t$. For functional differential equations without impulses, similar results are proved by Burton and Makay [3] using Lyapunov functionals.

\section{Prelimaries}

Let $r>0, \mathbb{R}^{s}$ be the $s$-dimensional Euclidean space with norm $\|\cdot\|, t_{0} \in \mathbb{R}_{+}=[0, \infty), J \subseteq \mathbb{R}$, and $\emptyset \neq D \subseteq \mathbb{R}^{s}$. Define the following class of functions:

$\mathrm{PC}[J, D]=\{\sigma: J \rightarrow D: \sigma$ is piecewise continuous with points of discontinuity $\tilde{t} \in J$ at which $\sigma(\tilde{t}-0)$ and $\sigma(\tilde{t}+0)$ exist and $\sigma(\tilde{t}-0)=\sigma(\tilde{t})\}$.

Let $\mathbb{R}_{H}^{s}=\left\{x \in \mathbb{R}^{s}:\|x\|<H\right\}$. Consider the system of impulsive functional differential equations with variable

\footnotetext{
*Corresponding author e-mail: bohner@mst.edu
} 
impulsive perturbations

$$
\left\{\begin{array}{c}
\dot{x}(t)=f\left(t, x_{t}\right)+g\left(t, x_{t}\right), \quad \dot{y}(t)=h\left(t, x_{t}, y_{t}\right), \\
t \neq \tau_{k}(x(t), y(t)), k \in \mathbb{N}, \\
\Delta x(t)=A_{k}(x(t))+B_{k}(y(t)), \\
t=\tau_{k}(x(t), y(t)), k \in \mathbb{N}, \\
\Delta y(t)=C_{k}(x(t), y(t)), \\
t=\tau_{k}(x(t), y(t)), k \in \mathbb{N},
\end{array}\right.
$$

where

$$
\begin{gathered}
f:\left[t_{0}, \infty\right) \times \mathrm{PC}\left[[-r, 0], \mathbb{R}_{H}^{n}\right] \rightarrow \mathbb{R}^{n}, \\
g:\left[t_{0}, \infty\right) \times \mathrm{PC}\left[[-r, 0], \mathbb{R}_{H}^{m}\right] \rightarrow \mathbb{R}^{n}, \\
h:\left[t_{0}, \infty\right) \times \mathrm{PC}\left[[-r, 0], \mathbb{R}_{H}^{n}\right] \times \mathrm{PC}\left[[-r, 0], \mathbb{R}_{H}^{m}\right] \rightarrow \mathbb{R}^{m},
\end{gathered}
$$

and for $k \in \mathbb{N}$,

$$
\begin{gathered}
\tau_{k}: \mathbb{R}_{H}^{n} \times \mathbb{R}_{H}^{m} \rightarrow\left(t_{0}, \infty\right), \quad A_{k}: \mathbb{R}_{H}^{n} \rightarrow \mathbb{R}^{n}, \\
B_{k}: \mathbb{R}_{H}^{m} \rightarrow \mathbb{R}^{n}, \quad C_{k}: \mathbb{R}_{H}^{n} \times \mathbb{R}_{H}^{m} \rightarrow \mathbb{R}^{m},
\end{gathered}
$$

$\Delta x(t)=x(t+0)-x(t-0), \Delta y(t)=y(t+0)-y(t-0)$, and for $t \geq t_{0}, x_{t} \in \mathrm{PC}\left[[-r, 0], \mathbb{R}_{H}^{n}\right]$ and $y_{t} \in \mathrm{PC}\left[[-r, 0], \mathbb{R}_{H}^{m}\right]$ are defined by

$$
x_{t}(s)=x(t+s) \quad \text { and } \quad y_{t}(s)=y(t+s)
$$

for $-r \leq s \leq 0$, respectively. Let

$$
\varphi_{0} \in \mathrm{PC}\left[[-r, 0], \mathbb{R}_{H}^{n}\right] \quad \text { and } \quad \phi_{0} \in \mathrm{PC}\left[[-r, 0], \mathbb{R}_{H}^{m}\right] .
$$

Denote by $(x, y)=\left(x\left(\cdot ; t_{0}, \varphi_{0}, \phi_{0}\right), y\left(\cdot ; t_{0}, \varphi_{0}, \phi_{0}\right)\right)$ the solution of system (1) satisfying the initial conditions

$$
\left\{\begin{array}{l}
x\left(t ; t_{0}, \varphi_{0}, \phi_{0}\right)=\varphi_{0}\left(t-t_{0}\right), \quad t_{0}-r \leq t \leq t_{0} \\
y\left(t ; t_{0}, \varphi_{0}, \phi_{0}\right)=\phi_{0}\left(t-t_{0}\right), \quad t_{0}-r \leq t \leq t_{0} \\
x\left(t_{0}+0 ; t_{0}, \varphi_{0}, \phi_{0}\right)=\varphi_{0}(0) \\
y\left(t_{0}+0 ; t_{0}, \varphi_{0}, \phi_{0}\right)=\phi_{0}(0)
\end{array}\right.
$$

The solutions $(x, y)$ of system (1) are piecewise continuous functions with points of discontinuity of the first kind in which they are left continuous (see [2]), i.e., at the moments $t_{l_{k}}$ when the integral curve of the solution $(x, y)$ meets the hypersurfaces

$$
\sigma_{k}=\left\{(t, x, y) \in\left[t_{0}, \infty\right) \times \mathbb{R}_{H}^{n} \times \mathbb{R}_{H}^{m}: t=\tau_{k}(x, y)\right\},
$$

the following relations are satisfied:

$$
\begin{gathered}
x\left(t_{l_{k}}-0\right)=x\left(t_{l_{k}}\right), \\
x\left(t_{l_{k}}+0\right)=x\left(t_{l_{k}}\right)+A_{l_{k}}\left(x\left(t_{l_{k}}\right)\right)+B_{l_{k}}\left(y\left(t_{l_{k}}\right)\right), \\
y\left(t_{l_{k}}-0\right)=y\left(t_{l_{k}}\right), \\
y\left(t_{l_{k}}+0\right)=y\left(t_{l_{k}}\right)+C_{l_{k}}\left(x\left(t_{l_{k}}\right), y\left(t_{l_{k}}\right)\right) .
\end{gathered}
$$

The points $t_{k}\left(t_{0}<t_{k}<t_{k+1}\right), k \in \mathbb{N}$, are the impulsive moments. Let us note that, in general, $k \neq l_{k}$. In other words, it is possible that the integral curve of the problem under consideration does not meet the hypersurface $\sigma_{k}$ at the moment $t_{k}$. It is clear that the solutions of systems with variable impulsive perturbations have points of discontinuity depending on the solutions, i.e., different solutions have different points of discontinuity. This leads to a number of difficulties in the investigation of such systems. One of the phenomena occurring with systems of type (1) is the so called "beating" of the solutions. This is the phenomenon when the mapping point $(t, x(t), y(t))$ meets one and the same hypersurface $\sigma_{k}$ several or infinitely many times $[2,7,8]$.

Together with system (1), we consider the impulsive system

$$
\left\{\begin{array}{l}
\dot{x}(t)=f\left(t, x_{t}\right), \quad t \neq \tau_{k}(x(t), 0), k \in \mathbb{N}, \\
\Delta x(t)=A_{k}(x(t)), \quad t=\tau_{k}(x(t), 0), k \in \mathbb{N} .
\end{array}\right.
$$

Let $\tau_{0}(x, y) \equiv t_{0}$ for $(x, y) \in \mathbb{R}_{H}^{n} \times \mathbb{R}_{H}^{m}$. We assume that the functions $\tau_{k}$ are continuous such that

$$
t_{0}<\tau_{1}(x, y)<\tau_{2}(x, y)<\ldots \tau_{k}(x, y) \rightarrow \infty \quad \text { as } \quad k \rightarrow \infty
$$

uniformly on $\mathbb{R}_{H}^{n} \times \mathbb{R}_{H}^{m}$. We also suppose that the functions $f, g, h, A_{k}, B_{k}$ and $C_{k}$ are smooth enough to guarantee existence, uniqueness, and continuability of the solution $(x, y)=\left(x\left(\cdot ; t_{0}, \varphi_{0}, \phi_{0}\right), y\left(\cdot ; t_{0}, \varphi_{0}, \phi_{0}\right)\right)$ of $(1)$ and of the solution $x\left(\cdot ; t_{0}, \varphi_{0}\right)$ of (3) for each $\varphi_{0} \in \mathrm{PC}\left[[-r, 0], \mathbb{R}_{H}^{n}\right], \phi_{0} \in \mathrm{PC}\left[[-r, 0], \mathbb{R}_{H}^{m}\right]$ and $t \geq t_{0}$. Existence and uniqueness criteria are given in $[2,8]$, and we have not included them here.

In the remainder of this paper, we shall use the following assumptions:

$\left(\mathrm{A}_{1}\right)$ There exists a constant $L>0$ such that

$$
\begin{gathered}
\left\|h\left(t, x_{t}, y_{t}\right)\right\| \leq L \text { for } \\
\left(t, x_{t}, y_{t}\right) \in\left[t_{0}, \infty\right) \times \mathrm{PC}\left[[-r, 0], \mathbb{R}_{H}^{n}\right] \times \mathrm{PC}\left[[-r, 0], \mathbb{R}_{H}^{m}\right] .
\end{gathered}
$$

$\left(\mathrm{A}_{2}\right)$ There exists a continuous function $P: \mathbb{R}_{+} \rightarrow \mathbb{R}_{+}$such that $P(0)=0$ and

$$
\|g(t, \phi(t))\| \leq P(\|\phi(t)\|) \quad \text { for } \quad t \in\left[t_{0}, \infty\right)
$$

and any function $\phi \in \mathrm{PC}\left[[t-r, t], \mathbb{R}_{H}^{m}\right]$.

$\left(\mathrm{A}_{3}\right)$ If $x \in \mathbb{R}_{H}^{n}$ and $y \in \mathbb{R}_{H}^{m}$, then for all $k \in \mathbb{N}$,

$$
\left\|x+A_{k}(x)+B_{k}(y)\right\| \leq\|x\|
$$

and

$$
\left\|y+C_{k}(x, y)\right\| \leq\|y\| .
$$

$\left(\mathrm{A}_{4}\right)$ The integral curves of system (1) meet successively each one of the hypersurfaces $\sigma_{k}, k \in \mathbb{N}$, at most once. $\left(\mathrm{A}_{5}\right) f(t, 0)=0, g(t, 0)=0, h(t, 0,0)=0$ for $t \in\left[t_{0}, \infty\right)$. $\left(\mathrm{A}_{6}\right) A_{k}(0)=0, B_{k}(0)=0, C_{k}(0,0)=0$ for $k \in \mathbb{N}$.

The condition $\left(\mathrm{A}_{4}\right)$ guarantees the absence of the phenomenon "beating" in (1). It is clear that in this case, the integral curve of each solution of system (3) meets each of the hypersurfaces

$$
s_{k}=\left\{(t, x) \in\left[t_{0}, \infty\right) \times \mathbb{R}_{H}^{n}: t=\tau_{k}(x, 0)\right\}
$$


at most once; i.e., for system (3), the phenomenon "beating" is not observed either. We point out that efficient sufficient conditions which guarantee the absence of "beating" of the solutions of impulsive functional differential systems are given in [2].

We now introduce the following notations:

$$
\begin{gathered}
\|\varphi\|_{r}=\sup _{t \in\left[t_{0}-r, t_{0}\right]}\left\|\varphi\left(t-t_{0}\right)\right\| \text { for } \varphi \in \operatorname{PC}\left[[-r, 0], \mathbb{R}_{H}^{n}\right], \\
\|\phi\|_{r}=\sup _{t \in\left[t_{0}-r, t_{0}\right]}\left\|\phi\left(t-t_{0}\right)\right\| \text { for } \phi \in \operatorname{PC}\left[[-r, 0], \mathbb{R}_{H}^{m}\right], \\
K=\left\{a \in \mathrm{C}\left[\mathbb{R}_{+}, \mathbb{R}_{+}\right]: a \uparrow, a(0)=0\right\},
\end{gathered}
$$

and for $k \in \mathbb{N}$,

$$
\begin{array}{r}
G_{k}=\left\{(t, x, y) \in\left[t_{0}, \infty\right) \times \mathbb{R}_{H}^{n} \times \mathbb{R}_{H}^{m}:\right. \\
\left.\tau_{k-1}(x, y)<t<\tau_{k}(x, y)\right\}, \\
\Omega_{k}=\left\{(t, x) \in\left[t_{0}, \infty\right) \times \mathbb{R}_{H}^{n} \times \mathbb{R}_{H}^{m}:\right. \\
\left.\tau_{k-1}(x, 0)<t<\tau_{k}(x, 0)\right\}
\end{array}
$$

and

$$
G=\bigcup_{k \in \mathbb{N}} G_{k}, \quad \Omega=\bigcup_{k \in \mathbb{N}} \Omega_{k} .
$$

We shall investigate the stability of the zero solution of system (1). To this end, we will use the following definitions of some stability properties of the zero solution of (1).

Definition 1. The zero solution of system (1) is said to be

(a) stable if for all $t_{0} \in \mathbb{R}_{+}$and for all $\varepsilon>0$, there exists $\delta=\delta\left(t_{0}, \varepsilon\right)>0$ such that for all

$$
\left(\varphi_{0}, \phi_{0}\right) \in \mathrm{PC}\left[[-r, 0], \mathbb{R}_{H}^{n}\right] \times \mathrm{PC}\left[[-r, 0], \mathbb{R}_{H}^{m}\right]
$$

satisfying

$$
\left\|\varphi_{0}\right\|_{r}+\left\|\phi_{0}\right\|_{r}<\delta
$$

we have

$$
\left\|x\left(t ; t_{0}, \varphi_{0}, \phi_{0}\right)\right\|+\left\|y\left(t ; t_{0}, \varphi_{0}, \phi_{0}\right)\right\|<\varepsilon
$$

for all $t \geq t_{0}$;

(b) uniformly stable if the number $\delta$ in (a) is independent of $t_{0} \in \mathbb{R}_{+}$;

(c) attractive if for all $t_{0} \in \mathbb{R}_{+}$, there exists $\lambda=\lambda\left(t_{0}\right)>0$ such that for all $\varepsilon>0$, for all

$$
\left(\varphi_{0}, \phi_{0}\right) \in \mathrm{PC}\left[[-r, 0], \mathbb{R}_{H}^{n}\right] \times \mathrm{PC}\left[[-r, 0], \mathbb{R}_{H}^{m}\right]
$$

satisfying

$$
\left\|\varphi_{0}\right\|_{r}+\left\|\phi_{0}\right\|_{r}<\lambda
$$

there exists $\gamma=\gamma\left(t_{0}, \varphi_{0}, \phi_{0}, \varepsilon\right)>0$ with

$$
\left\|x\left(t ; t_{0}, \varphi_{0}, \phi_{0}\right)\right\|+\left\|y\left(t ; t_{0}, \varphi_{0}, \phi_{0}\right)\right\|<\varepsilon
$$

for all $t \geq t_{0}+\gamma$; (d) equi-attractive if the number $\gamma$ in $(c)$ is independent of

$$
\left(\varphi_{0}, \phi_{0}\right) \in \mathrm{PC}\left[[-r, 0], \mathbb{R}_{H}^{n}\right] \times \mathrm{PC}\left[[-r, 0], \mathbb{R}_{H}^{m}\right] ;
$$

(e) uniformly attractive if the number $\lambda$ in (c) is independent of $t_{0} \in \mathbb{R}_{+}$and the number $\gamma$ in $(c)$ is independent of

$$
\left(t_{0}, \varphi_{0}, \phi_{0}\right) \in \mathbb{R}_{+} \times \mathrm{PC}\left[[-r, 0], \mathbb{R}_{H}^{n}\right] \times \mathrm{PC}\left[[-r, 0], \mathbb{R}_{H}^{m}\right]
$$

(f) asymptotically stable if it is stable and attractive;

(g) equi-asymptotically stable if it is stable and equi-attractive;

(h) uniformly asymptotically stable if it is uniformly stable and uniformly attractive.

Definition 2. A function $V:\left[t_{0}, \infty\right) \times \mathbb{R}_{H}^{n} \times \mathbb{R}_{H}^{m} \rightarrow \mathbb{R}$ belongs to class $V_{0}$ provided

1. $V$ is continuous in $G$ and locally Lipschitz continuous with respect to its second and third arguments on each of the sets $G_{k}, k \in \mathbb{N}$;

2. $V(t, 0,0)=0$ for $t \in\left[t_{0}, \infty\right)$;

3. For each $k \in \mathbb{N}$ and any point $\left(t_{0}^{*}, x_{0}^{*}, y_{0}^{*}\right) \in \sigma_{k}$, there exist the finite limits

$$
\begin{aligned}
& V\left(t_{0}^{*}-0, x_{0}^{*}, y_{0}^{*}\right)=\lim _{\substack{(t, x, y) \rightarrow\left(t_{0}^{*}, x_{0}^{*}, y_{0}^{*}\right) \\
(t, x, y) \in G_{k}}} V(t, x, y), \\
& V\left(t_{0}^{*}+0, x_{0}^{*}, y_{0}^{*}\right)=\lim _{\substack{(t, x, y) \rightarrow\left(t_{0}^{*}, x_{0}^{*}, y_{0}^{*}\right) \\
(t, x, y) \in G_{k+1}}} V(t, x, y),
\end{aligned}
$$

and the equality $V\left(t_{0}^{*}-0, x_{0}^{*}, y_{0}^{*}\right)=V\left(t_{0}^{*}, x_{0}^{*}, y_{0}^{*}\right)$ holds; 4. For each $k \in \mathbb{N}$ and any $(t, x, y) \in \sigma_{k}$, we have

$$
V\left(t+0, x+A_{k}(x)+B_{k}(y), y+C_{k}(x, y)\right) \leq V(t, x, y) .
$$

Definition 3. A function $W:\left[t_{0}, \infty\right) \times \mathbb{R}_{H}^{n} \rightarrow \mathbb{R}$ belongs to class $W_{0}$ provided

1. W is continuous in $\Omega$ and locally Lipschitz continuous with respect to its second argument on each of the sets $\Omega_{k}, k \in \mathbb{N}$;

2. $W(t, 0)=0$ for $t \in\left[t_{0}, \infty\right)$;

3. For each $k \in \mathbb{N}$ and any point $\left(t_{0}^{*}, x_{0}^{*}\right) \in s_{k}$, there exist the finite limits

$$
\begin{aligned}
& W\left(t_{0}^{*}-0, x_{0}^{*}\right)=\lim _{\substack{(t, x) \rightarrow\left(t_{0}^{*}, x_{0}^{*}\right) \\
(t, x) \in \Omega_{k}}} W(t, x), \\
& W\left(t_{0}^{*}+0, x_{0}^{*}\right)=\lim _{\substack{(t, x) \rightarrow\left(t_{0}^{*}, x_{0}^{*}\right) \\
(t, x) \in \Omega_{k+1}}} W(t, x),
\end{aligned}
$$

and the equality $W\left(t_{0}^{*}-0, x_{0}^{*}\right)=W\left(t_{0}^{*}, x_{0}^{*}\right)$ holds. 4. For each $k \in \mathbb{N}$ and any $(t, x) \in s_{k}$, we have

$$
W\left(t+0, x+A_{k}(x)\right) \leq W(t, x)
$$


Definition 4. Let $V \in V_{0}$. For $t \geq t_{0}$ with $t \neq \tau_{k}(x(t), y(t))$, $k \in \mathbb{N}$, and

$$
(\varphi, \phi) \in \mathrm{PC}\left[[t-r, t], \mathbb{R}_{H}^{n}\right] \times \mathrm{PC}\left[[t-r, t], \mathbb{R}_{H}^{m}\right],
$$

we define by

$$
\begin{gathered}
D_{(1)}^{+} V(t, \varphi(t), \phi(t))=\limsup _{\delta \rightarrow 0^{+}} \frac{1}{\delta}[-V(t, \varphi(t), \phi(t))+ \\
\left.V\left(t+\delta, \varphi(t)+\delta\left(f\left(t, \varphi_{t}\right)+g\left(t, \phi_{t}\right)\right), \phi(t)+\delta h\left(t, \varphi_{t}, \phi_{t}\right)\right)\right]
\end{gathered}
$$

the upper right-hand derivative of $V$ with respect to (1).

Note that in Definition $4, D_{(1)}^{+} V(t, \varphi(t), \phi(t))$ is a functional whereas $V$ is a function. This special feature was a source of difficulties in the application of the second method of Lyapunov for functional differential equations. Using simple considerations, Razumikhin [6] proved that the derivative $D_{(1)}^{+} V(t, \varphi(t), \phi(t))$ should be estimated only by the elements of minimal subsets of the integral curves of the investigated system when the condition

$$
V(t+s, \varphi(t+s), \phi(t+s))<V(t, \varphi(t), \phi(t)), s \in[-r, 0)
$$

holds. The condition (6) is called the Razumikhin condition, and the corresponding technique is known as Razumikhin technique [6-9].

Analogously, one can define the upper right-hand derivative $D_{(3)}^{+} W(t, \varphi(t))$ for an arbitrary function $W \in W_{0}$ for $t \geq t_{0}, t \neq \tau_{k}(x(t), 0), k \in \mathbb{N}$ and $\varphi \in \operatorname{PC}\left[[t-r, t], \mathbb{R}_{H}^{n}\right]$, which will be estimated whenever

$$
W(t+s, \varphi(t+s))<W(t, \varphi(t)), s \in[-r, 0) .
$$

Let $t_{k}<t_{k+1}, k \in \mathbb{N}$, be the moments in which the integral curve $\left(t, x\left(t ; t_{0}, \varphi_{0}, \phi_{0}\right), y\left(t ; t_{0}, \varphi_{0}, \phi_{0}\right)\right)$ of the problem (1), (2) meets the hypersurfaces $\sigma_{k}, k \in \mathbb{N}$. In the proof of the main results, we shall use the following lemma.

Lemma 1(See [8]). Assume that the function $V \in V_{0}$ is such that the inequality

$$
D^{+} V_{(1)}(t, \varphi(t), \phi(t)) \leq 0
$$

is valid for $t \in\left[t_{0}, \infty\right), t \neq t_{k}, k \in \mathbb{N}$, and any functions $(\varphi, \phi) \in \mathrm{PC}\left[[t-r, t], \mathbb{R}_{H}^{n}\right] \times \mathrm{PC}\left[[t-r, t], \mathbb{R}_{H}^{m}\right]$ for which (6) is true. Then, for $t \geq t_{0}$,

$$
\begin{aligned}
V\left(t, x\left(t ; t_{0}, \varphi_{0}, \phi_{0}\right), y\left(t ; t_{0}, \varphi_{0}, \phi_{0}\right)\right) & \\
& \leq V\left(t_{0}+0, \varphi_{0}(0), \phi_{0}(0)\right) .
\end{aligned}
$$

\section{Main Results}

Theorem 1. Assume the following.

1. Conditions $\left(A_{1}\right)-\left(A_{6}\right)$ hold.
2. There exist functions $V \in V_{0}$ and $a, c \in K$ such that

$$
\begin{aligned}
& a(\|x\|+\|y\|) \leq V(t, x, y), \\
& \quad \text { for all }(t, x, y) \in\left[t_{0}, \infty\right) \times \mathbb{R}_{H}^{n} \times \mathbb{R}_{H}^{m}
\end{aligned}
$$

and the inequality

$$
D_{(1)}^{+} V(t, \varphi(t), \phi(t)) \leq-c(\|\phi(t)\|)
$$

is valid for any $t \in\left[t_{0}, \infty\right), t \neq \tau_{k}(\varphi(t), \phi(t)), k \in \mathbb{N}$, and any functions

$$
(\varphi, \phi) \in \mathrm{PC}\left[[t-r, t], \mathbb{R}_{H}^{n}\right] \times \mathrm{PC}\left[[t-r, t], \mathbb{R}_{H}^{m}\right]
$$

that satisfy (6).

3. There exist functions $W \in W_{0}$ and $a_{1}, c_{1} \in K$ and $a$ constant $d>0$ such that

$$
a_{1}(\|x\| \mid) \leq W(t, x)
$$

for all $(t, x) \in\left[t_{0}, \infty\right) \times \mathbb{R}_{H}^{n}$,

$$
\left|W\left(t, x_{1}\right)-W\left(t, x_{2}\right)\right| \leq d\left\|x_{1}-x_{2}\right\|
$$

for all $t \in\left[t_{0}, \infty\right)$ and $x_{1}, x_{2} \in \mathbb{R}_{H}^{n}$, and the inequality

$$
D^{+} W_{(3)}(t, \varphi(t)) \leq-c_{1}(W(t, \varphi(t)))
$$

is valid for any $t \in\left[t_{0}, \infty\right), t \neq \tau_{k}(\varphi(t), 0) k \in \mathbb{N}$, and any function $\varphi \in \mathrm{PC}\left[[t-r, t], \mathbb{R}_{H}^{n}\right]$ that satisfies (7).

Then the zero solution of system (1) is asymptotically stable.

Proof. Let $\varepsilon \in(0, H)$ and $t_{0} \in \mathbb{R}_{+}$. From the condition $V\left(t_{0}, 0,0\right)=0$ and the properties of the function $V$, it follows that there exists a constant $\delta=\delta\left(t_{0}, \varepsilon\right)>0$ such that if $\|x\|+\|y\|<\delta$, then

$$
\sup _{\|x\|+\|y\|<\delta} V\left(t_{0}+0, x, y\right)<a(\varepsilon) .
$$

Let $\left(\varphi_{0}, \phi_{0}\right) \in \mathrm{PC}\left[[-r, 0], \mathbb{R}_{H}^{n}\right] \times \mathrm{PC}\left[[-r, 0], \mathbb{R}_{H}^{m}\right]$ be such that

$$
\left\|\varphi_{0}\right\|_{r}+\left\|\phi_{0}\right\|_{r}<\delta
$$

and let $(x, y)=\left(x\left(\cdot ; t_{0}, \varphi_{0}, \phi_{0}\right), y\left(\cdot ; t_{0}, \varphi_{0}, \phi_{0}\right)\right)$ be the solution of problem (1), (2). Since all conditions of Lemma 1 are met, we have

$$
\begin{aligned}
V\left(t, x\left(t ; t_{0}, \varphi_{0}, \phi_{0}\right), y\left(t ; t_{0}, \varphi_{0}, \phi_{0}\right)\right) & \\
& \leq V\left(t_{0}+0, \varphi_{0}(0), \phi_{0}(0)\right)
\end{aligned}
$$

for all $t \in\left[t_{0}, \infty\right)$. On the other hand,

$$
\left\|\varphi_{0}(0)\right\|+\left\|\phi_{0}(0)\right\| \leq\left\|\varphi_{0}\right\|_{r}+\left\|\phi_{0}\right\|_{r}<\delta
$$

and hence $V\left(t_{0}+0, \varphi_{0}(0), \phi_{0}(0)\right)<a(\varepsilon)$. From (8), (4) and the last inequality, we find

$a\left(\| x\left(t ; t_{0}, \varphi_{0}, \phi_{0}\|+\| y\left(t ; t_{0}, \varphi_{0}, \phi_{0}\right) \|\right)\right.$ 


$$
\begin{aligned}
& \leq V\left(t, x\left(t ; t_{0}, \varphi_{0}, \phi_{0}\right), y\left(t ; t_{0}, \varphi_{0}, \phi_{0}\right)\right) \\
& \leq V\left(t_{0}+0, \varphi_{0}(0), \phi_{0}(0)\right) \\
& <a(\varepsilon),
\end{aligned}
$$

which implies that

$$
\left\|x\left(t ; t_{0}, \varphi_{0}, \phi_{0}\right)\right\|+\left\|y\left(t ; t_{0}, \varphi_{0}, \phi_{0}\right)\right\|<\varepsilon
$$

for all $t \geq t_{0}$. This implies that the zero solution of system (1) is stable. Then we can choose a number $\lambda=\lambda\left(t_{0}\right)>0$ such that if

$$
\left\|\varphi_{0}\right\|_{r}+\left\|\phi_{0}\right\|_{r}<\lambda
$$

then

$$
\left\|x\left(t ; t_{0}, \varphi_{0}, \phi_{0}\right)\right\|+\left\|y\left(t ; t_{0}, \varphi_{0}, \phi_{0}\right)\right\|<H
$$

for any $t \geq t_{0}$. We shall prove that in this case

$$
\lim _{t \rightarrow \infty} y\left(t ; t_{0}, \varphi_{0}, \phi_{0}\right)=0 .
$$

If we suppose that (13) is not true, then for some $\varepsilon_{0}$, there exists a sequence $\left\{\xi_{R}\right\}_{R=1}^{\infty} \in\left[t_{0}, \infty\right)$ tending to $\infty$ for $R \rightarrow$ $\infty$ such that $\left\|y\left(\xi_{R}\right)\right\| \geq \varepsilon_{0}$ for all $R \in \mathbb{N}$. If $t_{k}, k \in \mathbb{N}$, are the moments in which the integral curve of the solution $\left(x\left(\cdot ; t_{0}, \varphi_{0}, \phi_{0}\right), y\left(\cdot ; t_{0}, \varphi_{0}, \phi_{0}\right)\right)$ meets the hypersurfaces $\sigma_{k}$, $k \in \mathbb{N}$, then for $t \neq t_{k}$, by $\left(\mathrm{A}_{1}\right)$, we obtain

$$
\left|\frac{\mathrm{d}}{\mathrm{d} t}\|y(t)\|\right| \leq\|\dot{y}(t)\|=\| h(t, x(t), y(t) \| \leq L
$$

We shall prove that

$$
\|y(t)\| \geq \varepsilon_{0} / 2 \quad \text { for } \quad t \in\left[\xi_{R}-\left(\varepsilon_{0} / 2 L\right), \xi_{R}\right]=J_{R} .
$$

In fact, let $0 \leq \xi_{R}-t \leq \varepsilon_{0} / 2 L$. Integrating (14) from $t$ to $\xi_{R}$, we obtain

$$
\int_{t}^{\xi_{R}} \frac{\mathrm{d}}{\mathrm{d} \tau}\|y(\tau)\| \mathrm{d} \tau \leq L\left(\xi_{R}-t\right) \leq \frac{\varepsilon_{0}}{2} .
$$

On the other hand, each interval $J_{R}, R \in \mathbb{N}$, contains a finite number of the points $\left\{t_{k}\right\}$. Assume, for instance, that these are the points $t_{s}, t_{s+1}, \ldots, t_{s+p}$. Then by $\left(\mathrm{A}_{3}\right)$, we obtain

$$
\begin{aligned}
\int_{t}^{\xi_{R}} \frac{\mathrm{d}}{\mathrm{d} \tau}\|y(\tau)\| \mathrm{d} \tau= & \int_{t}^{t_{s}} \frac{\mathrm{d}}{\mathrm{d} \tau}\|y(\tau)\| \mathrm{d} \tau \\
& +\sum_{j=s+1}^{s+p} \int_{t_{j-1}}^{t_{j}} \frac{\mathrm{d}}{\mathrm{d} \tau}\|y(\tau)\| \mathrm{d} \tau \\
& +\int_{t_{s+p}}^{\xi_{R}} \frac{\mathrm{d}}{\mathrm{d} \tau}\|y(\tau)\| \mathrm{d} \tau \\
= & \left\|y\left(t_{s}-0\right)\right\|-\|y(t+0)\| \\
& +\sum_{j=s+1}^{s+p}\left[\left\|y\left(t_{j}-0\right)\right\|-\left\|y\left(t_{j-1}+0\right)\right\|\right] \\
& +\left\|y\left(\xi_{R}-0\right)\right\|-\left\|y\left(t_{s+p}+0\right)\right\| \\
\geq & \left\|y\left(\xi_{R}\right)\right\|-\|y(t)\| .
\end{aligned}
$$

Therefore,

$\varepsilon_{0}-\|y(t)\| \leq\left\|y\left(\xi_{R}\right)\right\|-\|y(t)\| \leq \int_{t}^{\xi_{R}} \frac{\mathrm{d}}{\mathrm{d} \tau}\|y(\tau)\| \mathrm{d} \tau \leq \frac{\varepsilon_{0}}{2}$,

whence we deduce that $\|y(t)\| \geq \varepsilon_{0} / 2$. If we choose a suitable subsequence of the sequence $\left\{\xi_{R}\right\}$ (which we again denote by $\left\{\xi_{R}\right\}$ ), then we can assume that the intervals $J_{R}$ do not intersect one another and $t_{0}<\xi_{1}-\left(\varepsilon_{0} / 2 L\right)$. Then, from (9), we deduce that

$$
D_{(1)}^{+} V(t, \varphi(t), \phi(t)) \leq-c\left(\varepsilon_{0} / 2\right)
$$

in the intervals $J_{R}$ and

$$
D_{(1)}^{+} V(t, \varphi(t), \phi(t)) \leq 0
$$

for the remaining values of $t$ for which $t \neq \tau_{k}(\varphi(t), \phi(t))$, $k \in \mathbb{N}$, and whenever

$$
V(t+s, \varphi(t+s), \phi(t+s))<V(t, \varphi(t), \phi(t))
$$

for $s \in[-r, 0)$. Integrating and applying (4), we obtain

$$
\begin{aligned}
& V\left(\xi_{R}, x\left(\xi_{R}\right), y\left(\xi_{R}\right)\right) \leq V\left(t_{0}+0, \varphi_{0}(0), \phi_{0}(0)\right) \\
&-c\left(\frac{\varepsilon_{0}}{2}\right) \frac{\varepsilon_{0}}{L} R \rightarrow-\infty \quad \text { as } \quad R \rightarrow \infty,
\end{aligned}
$$

which contradicts (8). Hence, (13) holds. Next we shall prove that

$$
w(t)=W(t, x(t)) \rightarrow 0 \quad \text { as } \quad t \rightarrow \infty .
$$

Applying (11), we obtain

$$
D^{+} W_{(1)}(t, \varphi) \leq D^{+} W_{(3)}(t, \varphi)+d\|g(t, \phi)\|
$$

for $t \in\left[t_{0}, \infty\right), t \neq \tau_{k}(\varphi, \phi), k \in \mathbb{N}$, and for

$$
\varphi \in \mathrm{PC}\left[[t-r, t], \mathbb{R}_{H}^{n}\right], \quad \phi \in \mathrm{PC}\left[[t-r, t], \mid R_{H}^{m}\right] .
$$

Hence, by (12) and $\left(A_{2}\right)$, we have

$$
D^{+} W_{(1)}(t, \varphi(t)) \leq-c_{1}(W(t, \varphi(t)))+d P(\|\phi(t)\|)
$$

for $t \in\left[t_{0}, \infty\right), t \neq \tau_{k}(\varphi(t), \phi(t)), k \in \mathbb{N}$,

$$
\varphi \in \mathrm{PC}\left[[t-r, t], \mathbb{R}_{H}^{n}\right], \quad \phi \in \mathrm{PC}\left[[t-r, t], \mathbb{R}_{H}^{m}\right],
$$

and whenever $W(t+s, \varphi(t+s))<W(t, \varphi(t)), s \in[-r, 0)$. We set

$$
\limsup _{t \rightarrow \infty} w(t)=\alpha, \quad \liminf _{t \rightarrow \infty} w(t)=\beta .
$$

If we assume that $\alpha>\beta$, then for an arbitrarily small number $\mu>0$, we can find sequences $q_{n}>p_{n} \rightarrow \infty$ for $n \rightarrow \infty$ such that

$$
w\left(p_{n}\right)=\beta+\mu, \quad w\left(q_{n}\right)=\alpha-\mu,
$$


and $\beta+\mu<w(t)<\alpha-\mu$ for $p_{n}<t<q_{n}$. Since the function $P$ is continuous, $P(0)=0$, and $\lim _{t \rightarrow \infty} y(t)=0$, there exists $v \in \mathbb{N}$ such that for $n \geq v$ and $t \geq p_{n}$, we have

$$
P(\|\phi(t)\|) \leq \frac{c_{1}(\beta+\mu)}{d} .
$$

Then, from (16), we find

$$
D^{+} W_{(1)}(t, \varphi(t)) \leq-c_{1}(\beta+\mu)+d \frac{c_{1}(\beta+\mu)}{d}=0
$$

for $n \geq v$ and $t \in\left(p_{n}, q_{n}\right), t \neq \tau_{k}(\varphi(t), \phi(t))$, which together with (5) yields $w\left(p_{n}\right) \geq w\left(q_{n}\right)$. Hence $\beta+\mu \geq \alpha-\mu$, which contradicts the assumption that $\alpha>\beta$. This shows that there exists the limit

$$
\lim _{t \rightarrow \infty} W(t, x(t))=\sigma \geq 0 .
$$

If we assume now that $\sigma>0$, then we can find a number $T>0$ such that

$$
\frac{\sigma}{2} \leq W(t, \phi(t)) \leq \frac{3 \sigma}{2}
$$

and

$$
P(\|\phi(t)\|) \leq \frac{1}{2 d} c_{1}\left(\frac{\sigma}{2}\right)
$$

for all $t \geq T$. Then, applying (16) again, we obtain

$$
\begin{aligned}
D^{+} W_{(1)}(t, \varphi(t)) & \leq-c_{1}\left(\frac{\sigma}{2}\right)+d \frac{1}{2 d} c_{1}\left(\frac{\sigma}{2}\right) \\
& =-\frac{1}{2} c_{1}\left(\frac{\sigma}{2}\right)<0
\end{aligned}
$$

for $t \geq T$, so that by virtue of (5), through an integration, we get

$$
W(t, x(t))=W(T, x(T))-\frac{1}{2} c_{1}\left(\frac{\sigma}{2}\right)(t-T) \rightarrow-\infty
$$

as $t \rightarrow \infty$, which contradicts (10). Hence (15) holds. Thus, by (10), we obtain that

$$
\lim _{t \rightarrow \infty} x\left(t ; t_{0}, \varphi_{0}, \phi_{0}\right)=0 .
$$

Taking into account that $\lim _{t \rightarrow \infty} y\left(t ; t_{0}, \varphi_{0}, \phi_{0}\right)=0$, we conclude that the zero solution of system (1) is attractive. This completes the proof.

Theorem 2. Let the conditions of Theorem 1 be satisfied and let a function $b \in K$ exist such that

$$
V(t, x, y) \leq b(\|x\|+\|y\|),(t, x, y) \in\left[t_{0}, \infty\right) \times \mathbb{R}_{H}^{n} \times \mathbb{R}_{H}^{m} .
$$

Then the zero solution of system (1) is uniformly stable and equi-asymptotically stable.

Proof. Let $\varepsilon \in(0, H)$. Choose $\delta=\delta(\varepsilon)>0$ so that $b(\delta)<$ $a(\varepsilon)$. Let

$$
\left(\varphi_{0}, \phi_{0}\right) \in \mathrm{PC}\left[[-r, 0], \mathbb{R}_{H}^{n}\right] \times \mathrm{PC}\left[[-r, 0], \mathbb{R}_{H}^{m}\right]
$$

be such that

$$
\left\|\varphi_{0}\right\|_{r}+\left\|\phi_{0}\right\|_{r}<\delta
$$

and let $(x, y)=\left(x\left(\cdot ; t_{0}, \varphi_{0}, \phi_{0}\right), y\left(\cdot ; t_{0}, \varphi_{0}, \phi_{0}\right)\right)$ be the solution of problem (1), (2). As in Theorem 1, we prove that

$$
\begin{aligned}
& a\left(\| x\left(t ; t_{0}, \varphi_{0}, \phi_{0}\|+\| y\left(t ; t_{0}, \varphi_{0}, \phi_{0}\right) \|\right)\right. \\
& \leq V\left(t, x\left(t ; t_{0}, \varphi_{0}, \phi_{0}\right), y\left(t ; t_{0}, \varphi_{0}, \phi_{0}\right)\right) \\
& \leq V\left(t_{0}+0, \varphi_{0}(0), \phi_{0}(0)\right)
\end{aligned}
$$

for $t \geq t_{0}$. From the above inequalities and (17), we get the inequalities

$$
\begin{aligned}
& a\left(\| x\left(t ; t_{0}, \varphi_{0}, \phi_{0}\|+\| y\left(t ; t_{0}, \varphi_{0}, \phi_{0}\right) \|\right)\right. \\
& \leq V\left(t_{0}+0, \varphi_{0}(0), \phi_{0}(0)\right) \\
& \leq b\left(\left\|\varphi_{0}(0)\right\|+\left\|\phi_{0}(0)\right\|\right) \\
& \leq b\left(\left\|\varphi_{0}\right\|_{r}+\left\|\phi_{0}\right\|_{r}\right) \\
& <b(\delta)<a(\varepsilon)
\end{aligned}
$$

from which it follows that

$$
\left\|x\left(t ; t_{0}, \varphi_{0}, \phi_{0}\right)\right\|+\left\|y\left(t ; t_{0}, \varphi_{0}, \phi_{0}\right)\right\|<\varepsilon \quad \text { for } t \geq t_{0} .
$$

This proves the uniform stability of the zero solution of system (1). We can prove the equi-attractivity of the zero solution of system (1) by arguments analogous to those in the proof of Theorem 1 .

Theorem 3. Let the conditions of Theorem 2 be fulfilled and let a function $b_{1} \in K$ exist such that

$$
W(t, x) \leq b_{1}(\|x\|),(t, x) \in\left[t_{0}, \infty\right) \times \mathbb{R}_{H}^{n} .
$$

Then the zero solution of system (1) is uniformly asymptotically stable.

Proof. From Theorem 2, it follows that the zero solution of system (1) is uniformly stable. Hence, for any $\varepsilon \in(0, H]$, there exists $\delta=\delta(\varepsilon)>0, \delta<\varepsilon$, so that if

$$
\left(\varphi_{0}, \phi_{0}\right) \in \mathrm{PC}\left[[-r, 0], \mathbb{R}_{H}^{n}\right] \times \mathrm{PC}\left[[-r, 0], \mathbb{R}_{H}^{m}\right]
$$

is such that

$$
\left\|\varphi_{0}\right\|_{r}+\left\|\phi_{0}\right\|_{r}<\delta
$$

then

$$
\left\|x\left(t ; t_{0}, \varphi_{0}, \phi_{0}\right)\right\|+\left\|y\left(t ; t_{0}, \varphi_{0}, \phi_{0}\right)\right\|<\varepsilon
$$

for all $t \geq t_{0}$. Now we shall prove that the zero solution of system (1) is uniformly attractive. Choose $\delta_{1}=\delta_{1}(\varepsilon)>0$ so that $\delta_{1}(\varepsilon)<\delta / 2$ and

$$
P(\eta)<\frac{1}{2 d} c_{1}\left(a_{1}\left(\frac{1}{2} \delta\right)\right) \text { for } \quad 0 \leq \eta \leq \delta_{1},
$$

where $d$ is the Lipschitz constant for the function $W$. Let, moreover, $T_{1}=T_{1}(\varepsilon)>0$ and $T_{2}=T_{2}(\varepsilon)>0$ be such that

$$
T_{1}(\varepsilon)>\frac{b(H)-a\left(\delta_{1} / 2\right)}{c\left(\delta_{1} / 2\right)}
$$


and

$$
T_{2}(\varepsilon)>\frac{2\left[b_{1}(H)-a_{1}(\delta / 2)\right]}{c_{1}\left(a_{1}(\delta / 2)\right)} .
$$

Let $v \in \mathbb{N}$ be such that

$$
b(H)-(v-1) \frac{\delta_{1} c\left(\delta_{1} / 2\right)}{2 L}<0 .
$$

Let $t_{0} \in \mathbb{R}_{+}$,

$$
\left(\varphi_{0}, \phi_{0}\right) \in \mathrm{PC}\left[[-r, 0], \mathbb{R}_{H}^{n}\right] \times \mathrm{PC}\left[[-r, 0], \mathbb{R}_{H}^{m}\right]
$$

be such that

$$
\left\|\varphi_{0}\right\|_{r}+\left\|\phi_{0}\right\|_{r}<\delta
$$

and let $(x, y)=\left(x\left(\cdot ; t_{0}, \varphi_{0}, \phi_{0}\right), y\left(\cdot ; t_{0}, \varphi_{0}, \phi_{0}\right)\right)$ be the solution of problem (1), (2). Assume that for all $t \in\left(t_{0}, t_{0}+T_{1}\right]$, the inequality $\|y(t)\| \geq \delta_{1} / 2$ holds. Then from (9), we obtain

$$
D_{(1)}^{+} V(t, \varphi(t), \phi(t)) \leq-c(\|\phi(t)\|) \leq-c\left(\frac{\delta_{1}}{2}\right)
$$

for $t \in\left(t_{0}, t_{0}+T_{1}\right], t \neq \tau_{k}(\varphi(t), \phi(t)), k \in \mathbb{N}$, and for

$$
(\varphi, \phi) \in \operatorname{PC}\left[[t-r, t], \mathbb{R}_{H}^{n}\right] \times \operatorname{PC}\left[[t-r, t], \mathbb{R}_{H}^{m}\right]
$$

satisfying (6). Integrating (23) from $t_{0}$ to $t_{0}+T_{1}$ and using (4), (8), (20), we obtain

$$
\begin{aligned}
a\left(\frac{\delta_{1}}{2}\right) & \leq a\left(\left\|\varphi\left(t_{0}+T_{1}\right)\right\|+\left\|\phi\left(t_{0}+T_{1}\right)\right\|\right) \\
& \leq V\left(t_{0}+T_{1}, \varphi\left(t_{0}+T_{1}\right), \phi\left(t_{0}+T_{1}\right)\right) \\
& \leq V\left(t_{0}+0, \varphi_{0}(0), \phi_{0}(0)\right)-T_{1} c\left(\frac{\delta_{1}}{2}\right) \\
& <b(H)-c\left(\frac{\delta_{1}}{2}\right) \frac{b(H)-a\left(\delta_{1} / 2\right)}{c\left(\delta_{1} / 2\right)} \\
& =a\left(\frac{\delta_{1}}{2}\right) .
\end{aligned}
$$

The contradiction obtained shows that there exists $\xi_{1} \in\left(0, t_{0}+T_{1}\right]$ such that $\left\|y\left(\xi_{1}\right)\right\|<\delta_{1} / 2$. We shall prove that if for any $t \in\left[\xi_{1}, t_{0}+T_{1}+T_{2}\right]$, the inequality $\|y(t)\|<\delta_{1}$ holds, then there exists $\xi_{2} \in\left[\xi_{1}, t_{0}+T_{1}+T_{2}\right]$ such that $\left\|x\left(\xi_{2}\right)\right\|<\delta / 2$. Indeed, suppose that this is not true, i.e., for any $t \in\left[\xi_{1}, t_{0}+T_{1}+T_{2}\right]$, we have $\|x(t)\| \geq \delta / 2$. Then, by (12), ( $\left.\mathrm{A}_{2}\right)$, (19), and (10), we obtain

$$
\begin{aligned}
D^{+} W_{(1)}(t, \varphi(t)) & \leq D^{+} W_{(3)}(t, \varphi(t))+d\|g(t, \phi(t))\| \\
& \leq-c_{1}(W(t, \varphi(t)))+d P(\|\phi(t)\|) \\
& \leq-c_{1}\left(a_{1}(\|\varphi(t)\|)\right)+\frac{1}{2} c_{1}\left(a_{1}\left(\frac{\delta}{2}\right)\right) \\
& \leq-\frac{1}{2} c_{1}\left(a_{1}\left(\frac{\delta}{2}\right)\right)
\end{aligned}
$$

for $t \in\left[\xi_{1}, t_{0}+T_{1}+T_{2}\right], t \neq \tau_{k}(\varphi(t), \phi(t)), k \in \mathbb{N}$. Integrating (24) from $\xi_{1}$ to $t_{0}+T_{1}+T_{2}$ and using (10), (5), (18), and (21), we obtain

$a_{1}\left(\frac{\delta}{2}\right) \leq a_{1}\left(\left\|\varphi\left(t_{0}+T_{1}+T_{2}\right)\right\|\right)$

$$
\begin{aligned}
\leq & W\left(t_{0}+T_{1}+T_{2}, \varphi\left(t_{0}+T_{1}+T_{2}\right)\right) \\
\leq & W\left(\xi_{1}+0, \varphi_{0}\left(\xi_{1}\right)\right) \\
& -\frac{1}{2} c_{1}\left(a_{1}\left(\frac{\delta}{2}\right)\right)\left(t_{0}+T_{1}+T_{2}-\xi_{1}\right) \\
\leq & W\left(\xi_{1}+0, \varphi_{0}\left(\xi_{1}\right)\right)-\frac{1}{2} c_{1}\left(a_{1}\left(\frac{\delta}{2}\right)\right) T_{2} \\
< & b_{1}(H)-\frac{1}{2} c_{1}\left(a_{1}\left(\frac{\delta}{2}\right)\right) \frac{2\left[b_{1}(H)-a_{1}(\delta / 2)\right]}{c_{1}\left(a_{1}(\delta / 2)\right)} \\
= & a_{1}\left(\frac{\delta}{2}\right) .
\end{aligned}
$$

The contradiction obtained shows that there exists $\xi_{2} \in\left[\xi_{1}, t_{0}+T_{1}+T_{2}\right]$ such that $\left\|x\left(\xi_{2}\right)\right\|<\delta / 2$. Then we have

$$
\left\|x\left(\xi_{2}\right)\right\|+\left\|y\left(\xi_{2}\right)\right\|<\frac{\delta}{2}+\delta_{1}<\delta,
$$

and from the uniform stability, it follows that $\|x(t)\|+\|y(t)\|<\varepsilon$ for $t>\xi_{2}$. Hence $\|x(t)\|+\|y(t)\|<\varepsilon$ for $t \geq t_{0}+T_{1}(\varepsilon)+T_{2}(\varepsilon)$. Now, let us suppose that that there exists $\xi_{3} \in\left[\xi_{1}, t_{0}+T_{1}+T_{2}\right]$ for which $\left\|y\left(\xi_{3}\right)\right\| \geq \delta_{1}$ and let

$$
\xi_{5}=\inf \left\{t \in\left[\xi_{1}, t_{0}+T_{1}+T_{2}\right]:\|y(t)\| \geq \delta_{1}\right\} .
$$

If $\xi_{5}=\tau_{R}\left(x\left(\xi_{5}\right), y\left(\xi_{5}\right)\right)$ for some $R \in \mathbb{N}$, then $\left\|y\left(\xi_{5}+0\right)\right\|=\delta_{1}$ and $\left\|y\left(\xi_{5}\right)\right\|<\delta_{1}$. But then, from $\left(\mathrm{A}_{3}\right)$, we obtain that

$$
\begin{aligned}
\left\|y\left(\xi_{5}+0\right)\right\| & =\left\|y\left(\xi_{5}\right)+C_{R}\left(x\left(\xi_{5}\right), y\left(\xi_{5}\right)\right)\right\| \\
& \leq\left\|y\left(\xi_{5}\right)\right\|<\delta_{1} .
\end{aligned}
$$

The contradiction obtained shows that $\left\|y\left(\xi_{5}\right)\right\|=\delta_{1}$ and $\xi_{5} \neq \tau_{k}\left(x\left(\xi_{5}\right), y\left(\xi_{5}\right)\right)$ for $k \in \mathbb{N}$. Using again $\left(\mathrm{A}_{3}\right)$, we obtain that there exists $\xi_{4}$ with

$$
\xi_{1}<\xi_{4}<\xi_{5}<t_{0}+T_{1}+T_{2}
$$

such that $\xi_{4} \neq \tau_{k}\left(x\left(\xi_{4}\right), y\left(\xi_{4}\right)\right)$ for $k \in \mathbb{N},\left\|y\left(\xi_{4}\right)\right\|=\delta_{1} / 2$ and $\delta_{1} / 2<\|y(t)\|<\delta_{1}$ for $t \in\left(\xi_{4}, \xi_{5}\right)$. From assumption $\left(\mathrm{A}_{1}\right)$, it follows that $(\mathrm{d} / \mathrm{d} t)\|y(t)\| \leq L$ for $t \neq \tau_{k}(x(t), y(t))$, $k \in \mathbb{N}$, so that, as in the proof of Theorem 1 , we obtain that $\xi_{5}-\xi_{4} \geq \delta_{1} / 2 L$. From the choice of $\xi_{5}$, it is clear that

$$
V(s, \varphi(s), \phi(s))<V(t, \varphi(t), \phi(t))
$$

for $\xi_{4} \leq s \leq \xi_{5}$. Then from (9), we have

$$
D_{(1)}^{+} V(t, \varphi(t), \phi(t)) \leq-c(\|\phi(t)\|) \leq c\left(\frac{\delta_{1}}{2}\right)
$$

for $t \in\left[\xi_{4}, \xi_{5}\right], t \neq \tau_{k}(\varphi(t), \phi(t)), k \in \mathbb{N}$. Integrating (25) and using (4), we obtain

$$
\begin{aligned}
& V\left(\xi_{5}, \varphi\left(\xi_{5}\right), \phi\left(\xi_{5}\right)\right) \\
& \leq V\left(\xi_{4}, \varphi\left(\xi_{4}\right), \phi\left(\xi_{4}\right)\right)-c\left(\frac{\delta_{1}}{2}\right)\left(\xi_{5}-\xi_{4}\right) \\
& \leq V\left(\xi_{4}, \varphi\left(\xi_{4}\right), \phi\left(\xi_{4}\right)\right)-c\left(\frac{\delta_{1}}{2}\right) \frac{\delta_{1}}{2 L} .
\end{aligned}
$$

Thus we have proved that if $\left\|\varphi_{0}\right\|_{r}+\left\|\phi_{0}\right\|_{r}<\delta$, then exactly one of the following two cases is possible: 
1. $\left\|x\left(t ; t_{0}, \varphi_{0}, \phi_{0}\right)\right\|+\left\|y\left(t ; t_{0}, \varphi_{0}, \phi_{0}\right)\right\|<\varepsilon$ for

$$
t \geq t_{0}+T_{1}+T_{2} \text {. }
$$

2. There exist $\xi_{4}$ and $\xi_{5}$ with

$$
t_{0}<\xi_{4}<\xi_{5}<t_{0}+T_{1}+T_{2}
$$

such that

$$
\begin{aligned}
V\left(\xi_{5}, \varphi\left(\xi_{5}\right), \phi\left(\xi_{5}\right)\right) & \\
& \leq V\left(\xi_{4}, \varphi\left(\xi_{4}\right), \phi\left(\xi_{4}\right)\right)-c\left(\frac{\delta_{1}}{2}\right) \frac{\delta_{1}}{2 L}
\end{aligned}
$$

In the same way, we can prove that exactly one of following two possiblities takes place:

1. $\left\|x\left(t ; t_{0}, \varphi_{0}, \phi_{0}\right)\right\|+\left\|y\left(t ; t_{0}, \varphi_{0}, \phi_{0}\right)\right\|<\varepsilon$ for

$$
t \geq t_{0}+2\left[T_{1}+T_{2}\right] \text {. }
$$

2. There exist $\xi_{9}$ and $\xi_{10}$ with

$$
t_{0}+T_{1}+T_{2}<\xi_{9}<\xi_{10}<t_{0}+2\left[T_{1}+T_{2}\right]
$$

such that

$$
\begin{aligned}
& V\left(\xi_{10}, \varphi\left(\xi_{10}\right), \phi\left(\xi_{10}\right)\right) \\
& \quad \leq V\left(\xi_{9}, \varphi\left(\xi_{9}\right), \phi\left(\xi_{9}\right)\right)-c\left(\frac{\delta_{1}}{2}\right) \frac{\delta_{1}}{2 L} .
\end{aligned}
$$

By induction, we can prove that if $\left\|\varphi_{0}\right\|_{r}+\left\|\phi_{0}\right\|_{r}<\delta$, then we have exactly one of the following two cases:

1. $\left\|x\left(t ; t_{0}, \varphi_{0}, \phi_{0}\right)\right\|+\left\|y\left(t ; t_{0}, \varphi_{0}, \phi_{0}\right)\right\|<\varepsilon$ for

$$
t \geq t_{0}+(n-1)\left[T_{1}+T_{2}\right]
$$

2. There exist $\xi_{5 n-1}$ and $\xi_{5 n}$ with

$$
t_{0}+(n-1) T_{1}+T_{2}<\xi_{5 n-1}<\xi_{5 n}<t_{0}+n\left[T_{1}+T_{2}\right]
$$

such that

$$
\begin{aligned}
& V\left(\xi_{5 n}, \varphi\left(\xi_{5 n}\right), \phi\left(\xi_{5 n}\right)\right) \\
& \quad \leq V\left(\xi_{5 n-1}, \varphi\left(\xi_{5 n-1}\right), \phi\left(\xi_{5 n-1}\right)\right)-c\left(\frac{\delta_{1}}{2}\right) \frac{\delta_{1}}{2 L}
\end{aligned}
$$

If for any positive integer $n \geq v$ the second case holds, then, using

$$
\xi_{5(n-1)}<t_{0}+(n-1)\left[T_{1}+T_{2}\right]<\xi_{5 n-1},
$$

(9), and (22), we obtain

$$
\begin{aligned}
& V\left(\xi_{5 v}, \varphi\left(\xi_{5 v}\right), \phi\left(\xi_{5 v}\right)\right. \\
& \leq V\left(\xi_{5 v-1}, \varphi\left(\xi_{5 v-1}\right), \phi\left(\xi_{5 v-1}\right)\right)-c\left(\frac{\delta_{1}}{2}\right) \frac{\delta_{1}}{2 L} \\
& \leq V\left(\xi_{5(v-1)}, \varphi\left(\xi_{5(v-1)}\right), \phi\left(\xi_{5(v-1)}\right)\right)-c\left(\frac{\delta_{1}}{2}\right) \frac{\delta_{1}}{2 L}
\end{aligned}
$$

$$
\begin{aligned}
\leq & V\left(\xi_{5(v-1)-1}, \varphi\left(\xi_{5(v-1)-1}\right), \phi\left(\xi_{5(v-1)-1}\right)\right) \\
& -c\left(\frac{\delta_{1}}{2}\right) \frac{2 \delta_{1}}{2 L} \\
\leq & \ldots \\
\leq & V\left(\xi_{4}, \varphi\left(\xi_{4}\right), \phi\left(\xi_{4}\right)\right)-c\left(\frac{\delta_{1}}{2}\right) \frac{(v-1) \delta_{1}}{2 L}<0,
\end{aligned}
$$

which contradicts (8). Therefore, for

$$
t \geq t_{0}+v\left[T_{1}(\varepsilon)+T_{2}(\varepsilon)\right],
$$

we have

$$
\left\|x\left(t ; t_{0}, \varphi_{0}, \phi_{0}\right)\right\|+\left\|y\left(t ; t_{0}, \varphi_{0}, \phi_{0}\right)\right\|<\varepsilon .
$$

This completes the proof.

\section{An Example}

Let $x, y \in \mathbb{R}$ and $r>0$. Consider the impulsive system

$$
\left\{\begin{array}{c}
\dot{x}(t)=-p x(t)+q x(t-r)+q y(t), \\
t \neq \tau_{k}(x(t), y(t)), k \in \mathbb{N} \\
\dot{y}(t)=-q x(t)+p \sup _{s \in[-r, 0]} y(t+s)-\frac{p}{q} y(t), \\
t \neq \tau_{k}(x(t), y(t)), k \in \mathbb{N} \\
\Delta x(t)=a_{k} x(t), \quad \Delta y(t)=b_{k} x(t) \\
t=\tau_{k}(x(t), y(t)), k \in \mathbb{N}
\end{array}\right.
$$

where $q>0, p>0$, and for $k \in \mathbb{N}, a_{k}, b_{k} \in(-1,0]$ and

$$
\tau_{k}(x(t), y(t))=x^{2}(t)+y^{2}(t)+k .
$$

Then $\tau_{k} \in \mathrm{C}\left[\mathbb{R}^{2},(0, \infty)\right]$ for $k \in \mathbb{N}, \tau_{k}(x, y) \rightarrow \infty$ as $k \rightarrow \infty$ uniformly on $(x, y) \in \mathbb{R}^{2}$, and also

$$
0<\tau_{k}(x, y)<\tau_{k+1}(x, y) \quad \text { for } \quad(x, y) \in \mathbb{R}, k \in \mathbb{N} .
$$

Together with system (26), we consider the system

$$
\left\{\begin{array}{c}
\dot{x}(t)=-p x(t)+q x(t-r), \\
t \neq \tau_{k}(x(t), 0), k \in \mathbb{N}, \\
\Delta x(t)=a_{k} x(t), \\
t=\tau_{k}(x(t), 0), k \in \mathbb{N} .
\end{array}\right.
$$

Let $\varphi_{0}, \phi_{0} \in \mathrm{C}[[-r, 0], \mathbb{R}]$ and define

$$
V(t, x, y)=x^{2}+y^{2} \quad \text { and } \quad W(t, x)=x^{2} .
$$

Let $q \leq p$. Then for $t \geq 0$ and for any $\varphi, \phi \in \mathrm{PC}[[t-r, t], \mathbb{R}]$ such that

$$
V(t+s, \varphi(t+s), \phi(t+s))<V(t, \varphi(t), \phi(t))
$$

for $s \in[-r, 0)$, we have

$D_{(26)}^{+} V(t, \varphi(t), \phi(t))$ 


$$
\begin{aligned}
= & -2 p \varphi^{2}(t)+2 q \varphi(t) \varphi(t-r) \\
& +2 p \phi(t) \sup _{s \in[-r, 0]} \phi(t+s)-2 \frac{p}{q} \phi^{2}(t) \\
\leq & \left.q\left[\varphi^{2}(t)+\varphi^{2}(t-r)\right]+p\left[\phi^{2}(t)+\sup _{s \in[-r, 0]} \phi(t+s)\right)^{2}\right] \\
& -2 p \varphi^{2}(t)-2 \frac{p}{q} \phi^{2}(t) \\
\leq & 2 p\left[\varphi^{2}(t)+\phi^{2}(t)\right]-2 p \varphi^{2}(t)-2 \frac{p}{q} \phi^{2}(t) \\
= & -2 p\left(\frac{1}{q}-1\right) \phi^{2}(t)
\end{aligned}
$$

for $t \neq \tau_{k}(\varphi(t), \phi(t))$. Also,

$$
\begin{aligned}
D_{(27)}^{+} W(t, \varphi(t)) & =-2 p \varphi^{2}(t)+2 q \varphi(t) \varphi(t-r) \\
& \leq-2 p \varphi^{2}(t)+q\left[\varphi^{2}(t)+\varphi^{2}(t-r)\right] \\
& <2(-p+q) \varphi^{2}(t)
\end{aligned}
$$

for $t \geq 0$ and for any $\varphi \in \operatorname{PC}[[t-r, t], \mathbb{R}]$ such that

$$
W(t+s, \varphi(t+s))<W(t, \varphi(t)), s \in[-r, 0) .
$$

For $t=\tau_{k}(x(t), y(t)), k \in \mathbb{N}$, we have

$$
V\left(t+0, x(t)+a_{k} x(t)+b_{k} y(t)\right) \leq V(t, x(t), y(t)),
$$

and for $t=\tau_{k}(x(t), 0), k \in \mathbb{N}$, we have

$$
W\left(t+0, x(t)+a_{k} x(t)\right) \leq W(t, x(t)) .
$$

If there exists a constant $\alpha>0$ such that

$$
\frac{1}{q}-1 \geq \alpha>0,
$$

then all conditions of Theorem 3 are satisfied, and then the zero solution of (26) is uniformly asymptotically stable.

\section{References}

[1] J. O. Alzabut, G. Tr. Stamov, and E. Sermutlu. On almost periodic solutions for an impulsive delay logarithmic population model. Math. Comput. Modelling, 51, 625-631 (2010).

[2] Drumi Bainov and Angel Dishliev. The phenomenon "beating" of the solutions of impulsive functional-differential equations. Commun. Appl. Anal., 1, 435-441 (1997).

[3] T. A. Burton and G. Makay. Marachkov type stability results for functional-differential equations. Electron. $J$. Qual. Theory Differ. Equ., 17 (1998).

[4] Daniel Franco, Eduardo Liz, Juan J. Nieto, and Yuri V. Rogovchenko. A contribution to the study of functional differential equations with impulses. Math. Nachr., 218, 4960 (2000).

[5] Jack K. Hale and Sjoerd M. Verduyn Lunel. Introduction to functional-differential equations, Applied Mathematical Sciences. Springer-Verlag, New York, 99, (1993).

[6] B. S. Razumikhin. Ustoichivost ereditarnykh sistem. "Nauka", Moscow, (1988).

[7] Ivanka M. Stamova. Boundedness of impulsive functional differential equations with variable impulsive perturbations. Bull. Aust. Math. Soc., 77, 331-345 (2008).

[8] Ivanka M. Stamova. Stability analysis of impulsive functional differential equations, de Gruyter Expositions in Mathematics. Walter de Gruyter GmbH \& Co. KG, Berlin, 52, (2009).

[9] Qing Wang and Xinzhi Liu. Impulsive stabilization of delay differential systems via the Lyapunov-Razumikhin method. Appl. Math. Lett., 20, 839-845 (2007).

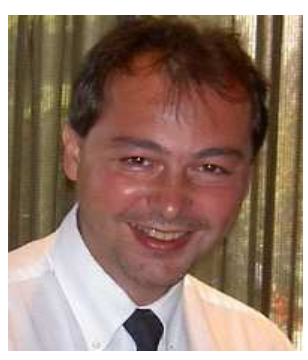

Martin Bohner is a Professor of Mathematics and Statistics at Missouri University of Science and Technology in Rolla, Missouri, USA. He received the BS (1989) and MS (1993) in Econo-mathematics and $\mathrm{PhD}$ (1995) from Universität Ulm, Germany, and MS (1992) in Applied Mathematics from San Diego State University. His research interests center around differential, difference, and dynamic equations as well as their applications to economics, finance, biology, physics, and engineering. He is the author of four textbooks and more than 180 publications, Editor-in-Chief of two international journals, Associate Editor for more than 50 international journals, and President of ISDE, the International Society of Difference Equations. Professor Bohner's honors at Missouri S\&T include five Faculty Excellence Awards, one Faculty Research Award, and eight Teaching Awards.

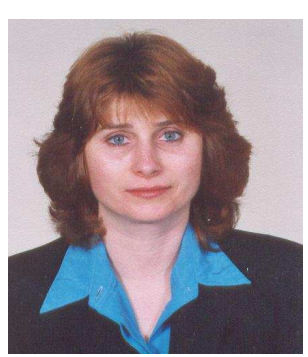

Ivanka Stamova received the Ph.D. degree in differential equations from the Higher Accreditation Commission of Bulgaria in 1996. In 2009, she received the Dr.Sci. degree in Applied Mathematics from the Higher Accreditation Commission of Bulgaria. She is the author of "Stability Analysis of Impulsive Functional Differential Equations", Walter de Gruyter, 2009, and an editor of "Lotka-Volterra and Related Systems: Recent Developments in Population Dynamics", Walter de Gruyter, 2013. A member of the AMS, she is serving in the Editorial Boards of several international mathematical and applied journals. Her current research interests include qualitative analysis of nonlinear dynamical systems, fractional differential systems, and impulsive control and applications. 